\title{
Large deviations and the equivalence of ensembles for Gibbsian particle systems with superstable interaction
}

\author{
Hans-Otto Georgii \\ Mathematisches Institut der Universität München, Theresienstrasse 39, D-80333 München, \\ Germany
}

Received: 2 June 1993/In revised form: 3 January 1994

Summary. For Gibbsian systems of particles in $\mathbf{R}^{d}$, we investigate large deviations of the translation invariant empirical fields in increasing boxes. The particle interaction is given by a superstable, regular pair potential. The large deviation principle is established for systems with free or periodic boundary conditions and, under a stronger stability hypothesis on the potential, for systems with tempered boundary conditions, and for tempered (infinite-volume) Gibbs measures. As a by-product we obtain the Gibbs variational formula for the pressure. We also prove the asymptotic equivalence of microcanonical and grand canonical Gibbs distributions and establish a variational expression for the thermodynamic entropy density.

Mathematics Subject Classification (1991): 60F10, 60G55, 60K35, 82B05, 82B21

\section{Introduction}

One of the classical themes of Equilibrium Statistical Mechanics is the study of the fluctuations of extensive quantities, such as the particle numbers and the energies of particle configurations in finite boxes, in the infinite-volume limit. This includes the problems of existence and variational characterization of the pressure, and of the asymptotic equivalence of the Gibbs ensembles on the level of measures. (A detailed discussion of these problems and their physical background can be found in the lectures of Lanford [11] and Martin-Löf [12].) For spin systems on a lattice, all these questions have been reconsidered successfully in the light of the recent progress in large deviation theory, see [8] and the references therein. In the case of continuous systems of point particles in Euclidean space, the situation is less satisfactory; the status so far is essentially still that set out by Lanford [11]. In this paper we develop a large deviation theory for such particle systems, with the aim of contributing to a systematic study of the questions above.

The general setting is as follows. We consider the Euclidean space $\mathbf{R}^{d}$ of any dimension $d \geqq 1$. A configuration of particles (without multiple occupancies) is de- 
scribed by a locally finite subset $\omega$ of $\mathbf{R}^{d}$, i.e., a set $\omega \subset \mathbf{R}^{d}$ having finite intersection with every bounded set. We write $\Omega$ for the set of all such configurations $\omega . \Omega$ is equipped with the $\sigma$-algebra $\mathcal{F}$ generated by the counting variables $N_{B}: \omega \rightarrow \operatorname{card}(\omega \cap B)$ for Borel subsets $B$ of $\mathbf{R}^{d}$. It is well-known [10,13] that $\mathcal{F}$ is the Borel $\sigma$-algebra for a natural Polish topology on $\Omega$. The translation group $\Theta=\left(\vartheta_{x}\right)_{x \in \mathbf{R}^{d}}$ acting on $(\Omega, \mathcal{F})$ is defined by $\vartheta_{x} \omega=\{y-x: y \in \omega\}, \omega \in \Omega, x \in \mathbf{R}^{d}$. The mapping $(x, \omega) \rightarrow \vartheta_{x} \omega$ is known to be measurable [13]. We let $\mathcal{P}$ denote the set of all probability measures $P$ on $(\Omega, \mathcal{F})$ with finite expected particle numbers $P\left(N_{\Delta}\right) \equiv \int N_{A} d P$ in bounded Borel sets $\Delta \subset \mathbf{R}^{d}$, and we write $\mathcal{P}_{\Theta}$ for the set of all $\Theta$-invariant $P \in \mathcal{P}$. For each $P \in \mathcal{P}_{\Theta}$ there exists a number $\rho(P)<\infty$, the intensity of $P$, such that $P\left(N_{\Delta}\right)=\rho(P)|\Delta|$ for all Borel sets $\Delta$. Here $|\Delta|$ is the Lebesgue measure of $\Delta$.

We introduce a topology $\tau_{\mathcal{L}}$ on $\mathcal{P}$ as follows. Let $\mathcal{L}$ denote the class of all measurable functions $f: \Omega \rightarrow \mathbf{R}$ which are local, in that $f(\omega)=f(\omega \cap \Delta)$ for some bounded Borel set $\Delta$ and all $\omega \in \Omega$, and tame, in that $|f| \leqq c\left(1+N_{A}\right)$ for (without loss the same) $\Delta$ and some constant $c<\infty$. The topology $\tau_{\mathcal{L}}$ of local convergence is then defined as the weak ${ }^{\star}$ topology on $\mathcal{P}$ relative to $\mathcal{L}$, i.e., as the smallest topology on $\mathcal{P}$ making the mappings $P \rightarrow P(f) \equiv \int f d P$ continuous. In particular, the mappings $P \rightarrow P\left(N_{A}\right)$ for bounded Borel sets $A$ are continuous relative to $\tau_{\mathcal{L}}$. This shows that $\tau_{\mathcal{L}}$ is much finer than the usual weak topology on $\mathcal{P}$ which is based on the above-mentioned Polish topology on $\Omega$.

The basic objects of interest for studying large deviations are the $\Theta$-invariant empirical fields in increasing boxes. ( For general information on concepts, use, and recent developments in large deviation theory we refer to the monographs [1, 2].) Thus, for each $n \geqq 0$ we consider the half-open cube $\Lambda_{n}=\left[-n-\frac{1}{2}, n+\frac{1}{2}{ }^{d}\right.$ of volume $v_{n}=(2 n+1)^{d}$ and the associated translation invariant empirical field

$$
R_{n, \omega}=v_{n}^{-1} \int_{\Lambda_{n}} \delta_{\vartheta_{x} \omega^{(n)}} d x
$$

of any configuration $\omega \in \Omega$. In (1.1), we replaced $\omega$ by the $A_{n}$-periodic continuation

$$
\omega^{(n)}=\left\{x+(2 n+1) i: x \in \omega \cap \Lambda_{n}, i \in \mathbf{Z}^{d}\right\}
$$

of its restriction to $A_{n}$. This has the advantage of making $R_{n, \omega}$ translation invariant. Thus $R_{n, \omega} \in \mathcal{P}_{\Theta}$ for all $n$ and $\omega$. If $\mathcal{P}_{\Theta}$ is equipped with the evaluation $\sigma$-algebra generated by the maps $P \rightarrow P(A), A \in \mathcal{F}$, the random measure $R_{n}: \omega \rightarrow R_{n, \omega}$ becomes a measurable mapping from $\Omega$ to $\mathcal{P}_{\Theta}$. The asymptotic behavior of the empirical fields can be described as follows: For each $P \in \mathcal{P}_{\Theta}$,

$$
\tau_{\mathcal{L}}-\lim _{n \rightarrow \infty} R_{n}=P_{\mathcal{I}} \quad \text { in } \quad P \text {-probability, }
$$

where $\omega \rightarrow P_{\mathcal{I}}^{\omega}$ is a regular conditional probability of $P$ relative to the $\sigma$-algebra $\mathcal{I}$ of $\Theta$-invariant sets in $\mathcal{F}$. This follows immediately from Wiener's multidimensional mean ergodic theorem, cf. [10].

In this paper we study large deviations from the ergodic theorem (1.2) when $P$ is Gibbsian relative to a suitable pair interaction $\varphi$. More precisely, we establish a large deviation principle for $R_{n}$ when the particles are distributed according to a Gibbs distribution in $\Lambda_{n}$ with free or periodic boundary condition and the underlying potential $\varphi$ is superstable and satisfies a decay condition called regularity (see Theorem 2). Under the stronger hypothesis that $\varphi$ diverges at the origin sufficiently fast, we obtain a uniform large deviation principle relative to Gibbs 
distributions with boundary configurations $\zeta \in \Omega$ satisfying a uniform condition of temperedness (Theorem 3(a)). By virtue of the well-known superstability estimates of Ruelle [18], this leads to a large deviation principle for tempered Gibbs measures on $(\Omega, \mathcal{F})$ (Theorem $3(\mathrm{~b})$ ). The rate function is, of course, given by the excess of the free energy density over its equilibrium value. In particular, we establish the Gibbs variational formula which asserts that this equilibrium value is given by the pressure (Eqs. (2.15) and (2.22)). A basic ingredient of all this is the existence and lower semicontinuity of the (internal) energy density (Theorem 1). Finally, we prove a limit theorem for conditional Poisson distributions of microcanonical type, implying the equivalence of Gibbs ensembles on the level of measures (Theorem 4). This is an instance of the maximum entropy principle and is closely related to a (microcanonical) Gibbs variational formula for the thermodynamic entropy density.

In the Poissonian case of no interaction, the analoguous results were obtained earlier in [10]. Our results here rely heavily on this paper. A weak version of a large deviation principle for particles with superstable interaction of finite range is also contained in [15]. The case of particles with hard core (which is contained in the present work) was already treated in [9].

\section{Statement of results}

We begin describing the particle interactions which we will consider. We assume, for simplicity, that the interaction is only pairwise and thus given by an even measurable function $\varphi: \mathbf{R}^{d} \rightarrow \mathbf{R} \cup\{\infty\}$. Such a $\varphi$ is called a potential. For each $n \geqq 0$,

$$
H_{n}(\omega) \equiv H_{n, \text { free }}(\omega)=\frac{1}{2} \sum_{x, y \in \omega \cap A_{n}, x \neq y} \varphi(y-x), \quad \omega \in \Omega,
$$

is called the associated Hamiltonian in $A_{n}$ with free boundary condition. A potential $\varphi$ is said to be stable if there exists a constant $b=b(\varphi)<\infty$ such that

$$
H_{n} \geqq-b N_{n} \text { for all } n \geqq 0 .
$$

Here $N_{n}=N_{\Lambda_{n}}$. In particular, (2.2) implies that $\varphi \geqq-2 b$. Sufficient conditions for $\varphi$ to be stable can be found in [17] . Let us say that $\varphi$ is purely repulsive if $\varphi$ is nonnegative and bounded away from zero near the origin, i.e., if there exists some $\delta=\delta(\varphi)>0$ such that

$$
\varphi \geqq \delta 1_{\{|\cdot| \leqq \delta\}} .
$$

Here and below, $|\cdot|$ stands for the maximum norm on $\mathbf{R}^{d}$. A potential $\varphi$ is called superstable if

$$
\varphi=\varphi^{s}+\varphi^{r}
$$

for a stable $\varphi^{s}$ and a purely repulsive $\varphi^{r}$. The use of this concept was revealed by the pioneering work of Ruelle $[16,18]$ (Related ideas appeared also in the independent work $[3,4]$.)

Besides the hypothesis of superstability which assures that large particle numbers in a bounded region require a large amount of energy we shall also need a condition on the decay of $\varphi$. A potential $\varphi$ is called lower regular if there exists a decreasing function $\psi:[0, \infty[\rightarrow[0, \infty[$ such that

$$
\varphi(x) \geqq-\psi(|x|) \text { for all } x \in \mathbf{R}^{d}
$$


and

$$
\int_{0}^{\infty} \psi(s) s^{d-1} d s<\infty .
$$

$\varphi$ will be called regular if $\varphi$ is lower regular and, in addition, there exists some $r(\varphi)<\infty$ such that

$$
\varphi(x) \leqq \psi(|x|) \quad \text { whenever } \quad|x| \geqq r(\varphi) .
$$

Our first result is the existence of the energy density of any $P \in \mathcal{P}_{\Theta}$. To state it we need to recall that the Palm measure of $P \in \mathcal{P}_{\Theta}$ is defined as the unique finite measure $P^{\circ}$ on $(\Omega, \mathcal{F})$ satisfying

$$
\int P(d \omega) \sum_{x \in \omega} f\left(x, \vartheta_{x} \omega\right)=\int d x \int P^{\circ}(d \omega) f(x, \omega)
$$

for all measurable functions $f: \mathbf{R}^{d} \times \Omega \rightarrow\left[0, \infty\left[\right.\right.$. We have $P^{\circ}(\Omega)=P^{\circ}(\{\omega \in \Omega$ : $\omega \ni 0\})=\rho(P)$, and the normalized Palm measure $\rho(P)^{-1} P^{\circ}$ can be viewed as the natural version of the conditional probability $P(\cdot \mid\{\omega \in \Omega: \omega \ni 0\})$; see [13] for more details. Let us introduce the set

$$
\mathcal{P}_{\Theta}^{(2)}=\left\{P \in \mathcal{P}_{\Theta}: P\left(N_{\Delta}^{2}\right)<\infty \text { for all bounded Borel sets } A\right\}
$$

of all second-order elements of $\mathcal{P}_{\Theta}$. For each $n \geqq 0$ and $\mathcal{P} \in \mathcal{P}_{\Theta}$ we let

$$
\Phi_{n}(P)=v_{n}^{-1} P\left(H_{n}\right),
$$

be the expected $\varphi$-energy per volume in $\Lambda_{n}$. In view of (2.2), $\Phi_{n}$ is well-defined (possibly equal to $+\infty$ ), and $\Phi_{n} \geqq-b \rho$. In Section 3 we shall prove the following.

Theorem 1 Suppose $\varphi$ is superstable and lower regular. Then, for each $P \in \mathcal{P}_{\Theta}$, the limit $\Phi(P)=\lim _{n \rightarrow \infty} \Phi_{n}(P)$ exists and satisfies

$$
\Phi(P)= \begin{cases}P^{\circ}\left(f_{\varphi}\right) & \text { if } P \in \mathcal{P}_{\Theta}^{(2)} \\ \infty & \text { otherwise }\end{cases}
$$

where

$$
f_{\varphi}(\omega)=\frac{1}{2} \sum_{0 \neq y \in \omega} \varphi(y), \quad \omega \in \Omega .
$$

Moreover, the function $\Phi: \mathcal{P}_{\Theta} \rightarrow \mathbf{R} \cup\{\infty\}$ is lower semicontinuous (relative to $\tau_{\mathcal{L}}$ ).

$\Phi(P)$ is called the energy density of $P$. It is clear that $\Phi$ is affine. To state our large deviation results we next need to introduce the entropy density. We let $Q \in \mathcal{P}_{\Theta}$ denote the Poisson point random field on $\mathbf{R}^{d}$ with intensity $\rho(Q)=1$. For each $P \in \mathcal{P}_{\Theta}$ and $n \geqq 0$ we write

$$
P_{n}=P\left(\left\{\omega \in \Omega: \omega \cap A_{n} \in \cdot\right\}\right)
$$

for the restriction of $P$ to $A_{n}$. We think of $P_{n}$ as an element of $\mathcal{P}$ which is supported on $\Omega_{n}=\left\{\omega \in \Omega: \omega \subset A_{n}\right\}$. The negative entropy density of $P$ is then defined as the (existing) limit

$$
I(P)=\lim _{n \rightarrow \infty} v_{n}^{-1} I\left(P_{n} ; Q_{n}\right),
$$


where $I(\cdot ; \cdot)$ stands for the relative entropy; see $[10]$ for more details. $I$ is an affine function with $\tau_{\mathcal{L}}$-compact level sets [10].

Suppose now we are given an inverse temperature $\beta>0$ and an activity $z>0$. (Later on, we shall assume without loss that the units are chosen so that $\beta=z=1$.) The excess free energy density of $P \in \mathcal{P}_{\Theta}$ is then given by

$$
I_{z, \beta}(P)=I(P)+\beta \Phi(P)-\rho(P) \log z+p(z, \beta),
$$

where

$$
p(z, \beta)=-\min [I+\beta \Phi-\rho \log z] .
$$

Theorem 2 below asserts that $p(z, \beta)$ is nothing other than ( $\beta$ times) the pressure, and $I_{z, \beta}$ is the rate function in a large deviation principle for the distribution of the empirical fields $R_{n}$ under the Gibbs distributions with free or periodic boundary conditions. (Configurational boundary conditions will be considered later in Theorem 3.)

For $n \geqq 0$, the Hamiltonian in $A_{n}$ with periodic boundary condition is defined by

$$
\begin{aligned}
H_{n, \mathrm{per}}(\omega) & =v_{n} \Phi\left(R_{n, \omega}\right) \\
& =\frac{1}{2} \sum_{x \in \omega \cap A_{n}, y \in \omega^{(n)}, y \neq x} \varphi(y-x) .
\end{aligned}
$$

The last equation follows from (2.11) and the easily verified fact that the Palm measure of $R_{n, \omega}$ is given by

$$
R_{n, \omega}^{\circ}=v_{n}^{-1} \sum_{x \in \omega \cap A_{n}} \delta_{\vartheta_{x} \omega^{(n)}},
$$

cf. [10]. For a given boundary condition bc $\in\{$ per, free $\}$, the associated Gibbs distribution in $A_{n}$ with parameters $z, \beta>0$ is defined by

$$
P_{n, z, \beta, \mathrm{bc}}(d \omega)=Z_{n, z, \beta, \mathrm{bc}}^{-1} z^{N_{n}(\omega)} \exp \left[-\beta H_{n, \mathrm{bc}}(\omega)\right] Q_{n}(d \omega)
$$

where

$$
Z_{n, z, \beta, \mathrm{bc}}=Q_{n}\left(z^{N_{n}} \exp \left[-\beta H_{n, \mathrm{bc}}\right]\right)
$$

is the so-called partition function. It follows from (2.2) resp. Theorem 1 and (3.3) below that $Z_{n, z, \beta, \text { bc }}$ is finite. Thus $P_{n, z, \beta \text {,bc }}$ is well-defined. Again, we think of it as an element of $\mathcal{P}$ with support $\Omega_{n}$.

Theorem 2 Let $\varphi$ be superstable and regular, $z, \beta>0$, and $F: \mathcal{P}_{\Theta} \rightarrow \mathbf{R} \cup\{\infty\}$ a measurable functional satisfying $F \geqq-c(1+\rho)$ for some $c<\infty$. Then, for bc $=$ per or free,

$$
\lim _{n \rightarrow \infty} \sup _{n}^{-1} \log P_{n, z, \beta, \mathrm{bc}}\left(e^{-v_{n} F\left(R_{n}\right)}\right) \leqq-\inf \left[I_{z, \beta}+F_{\mathrm{lsc}}\right]
$$

and

$$
\liminf _{n \rightarrow \infty} v_{n}^{-1} \log P_{n, z, \beta, \mathrm{bc}}\left(e^{-v_{n} F\left(R_{n}\right)}\right) \geqq-\inf \left[I_{z, \beta}+F^{\mathrm{usc}}\right],
$$

where $F_{\mathrm{lsc}}$ is the largest lower semicontinuous minorant and $F^{\mathrm{usc}}$ the lowest upper semicontinuous majorant of $F$ relative to $\tau_{\mathcal{L}}$. In addition, $I_{z, \beta}$ has $\tau_{\mathcal{L}}$-compact level sets, and 


$$
p(z, \beta)=\lim _{n \rightarrow \infty} v_{n}^{-1} \log Z_{n, z, \beta, \mathrm{bc}}
$$

Note that (2.20) and (2.21) take the familiar form of a large deviation principle when $F$ is chosen to be zero on some measurable set $A \subset \mathcal{P}_{\Theta}$ and equal to $+\infty$ outside $A$. The existence of the limit in (2.22) is the classical result on the existence of the pressure; p. 68 of [17] contains the relevant bibliographical notes. The coincidence of the right sides of (2.15) and (2.22) is called the Gibbs variational formula. (In the case of a hard-core interaction, this variational formula has already been proved in [6].) The upper bound (2.20) will be proved in Sect. 4 and the lower bound (2.21) in Sect. 5.

We now turn to large deviations for Gibbs distributions with configurational boundary conditions, and for infinite-volume Gibbs measures. We need some notations. Let $C=\left[-1 / 2,1 / 2\left[^{d}=\Lambda_{0}\right.\right.$ be the centered half-open unit cube and $L=\mathbf{Z}^{d}$. The sets $C+i, i \in L$, form a partition of $\mathbf{R}^{d}$. For $n \geqq 0$ we set $L_{n}=L \cap A_{n}=$ $\{i \in L:|i| \leqq n\}$ and

$$
T_{n}=\sum_{i \in L_{n}} N_{C+i}^{2}
$$

For $t>0$ we define

$$
\Omega(t)=\left\{T_{n} \leqq t v_{n} \text { for all } n \geqq 0\right\} .
$$

The configurations in $\Omega^{\star}=\bigcup_{t>0} \Omega(t)$ are called tempered. The multidimensional ergodic theorem shows that $P\left(\Omega^{\star}\right)=1$ for all $P \in \mathcal{P}_{\Theta}^{(2)}$.

For each $\zeta \in \Omega^{\star}$ and $n \geqq 0$ we let

$$
H_{n, \zeta}(\omega)=H_{n}(\omega)+\sum_{x \in \omega \cap A_{n}, y \in \zeta \backslash \Lambda_{n}} \varphi(y-x)
$$

denote the Hamiltonian in $\Lambda_{n}$ with boundary condition $\zeta$. The associated Gibbs distributions $P_{n, z, \beta, \zeta}$ are defined by (2.18) with bc $=\zeta$. By Lemma 4.2, the last sum in (2.25) exists when $\varphi$ is regular. Under the hypotheses of Theorem 3 below, Lemma 6.1 and the estimates in the proof of Lemma 6.3 even imply that $Z_{n, z, \beta, \zeta}<\infty$, so that $P_{n, z, \beta, \zeta}$ is well-defined.

A measure $P \in \mathcal{P}$ is called a tempered Gibbs measure for $z, \beta>0$ if $P\left(\Omega^{\star}\right)=1$ and, for all $n \geqq 0$ and measurable functions $f \geqq 0$ on $\Omega$,

$$
P(f)=\int P(d \zeta) \int P_{n, z, \beta, \zeta}(d \omega) f\left(\omega \cup\left(\zeta \backslash \Lambda_{n}\right)\right) .
$$

Note that the identity above is equivalent to the equilibrium equations in [18].

Let us say $\varphi$ is non-integrably divergent at the origin if there exists a decreasing function $\chi:] 0, \infty[\rightarrow[0, \infty[$ such that

$$
\varphi(x) \geqq \chi(|x|) \quad \text { whenever } \quad \varphi(x) \geqq 0
$$

and

$$
\int_{0}^{1} \chi(s) s^{d-1} d s=\infty
$$

Together with the lower regularity, this condition implies that $\varphi$ is superstable, see Proposition 3.2 .8 of [17]. It also follows that tempered Gibbs measures exist; this was proved independently in $[3,18]$. Under this hypothesis, the following theorem 
provides a uniform large deviation principle for Gibbs distributions with (uniformly) tempered boundary conditions, and a large deviation principle for tempered Gibbs measures. Its proof will be given in Sect. 6 .

Theorem 3 Suppose $\varphi$ is regular and non-integrably divergent at the origin. Also, let $F$ be as in Theorem 2 and $z, \beta>0$.

(a) For each $t>0$, we have

$$
\limsup _{n \rightarrow \infty} v_{n}^{-1} \log \sup _{\zeta \in \Omega(t)} P_{n, z, \beta, \zeta}\left(e^{-v_{n} F\left(R_{n}\right)}\right) \leqq-\inf \left[I_{z, \beta}+F_{\mathrm{lsc}}\right]
$$

and

$$
\liminf _{n \rightarrow \infty} v_{n}^{-1} \log _{\zeta \in \Omega(t)} \inf _{n, z, \beta, \zeta}\left(e^{-v_{n} F\left(R_{n}\right)}\right) \geqq-\inf \left[I_{z, \beta}+F^{\mathrm{usc}}\right] .
$$

Moreover, (2.22) holds with $\mathrm{bc}=\zeta$ uniformly for all $\zeta \in \Omega(t)$.

(b) Each tempered Gibbs measure $P$ with parameters $z, \beta$ satisfies a large deviation principle for $R_{n}$ with rate function $I_{z, \beta}$, in that inequalities (2.20) and (2.21) hold with $P$ instead of $P_{n, z, \beta, b \mathrm{c}}$.

Let us note that an application of the contraction principle to Theorems 2 and 3 leads to analoguous large deviation principles for the individual empirical fields $R_{n}^{\circ}$ in (2.17); see [10] for more details. We also note that, under the hypotheses of Theorem 3, the rate function $I_{z, \beta}$ vanishes precisely on the set of all $\Theta$-invariant tempered Gibbs measures with parameters $z, \beta$. One direction of this variational principle follows by standard arguments from Eq. (1.2) and the upper bound in Theorem 3 (b). The reverse direction can be obtained by an adaptation of the proof of the analogous result in the lattice case, see Theorem (15.37) in [7]. Details will be provided elsewhere.

Our last result is a version of the equivalence of ensembles. For any nondegenerate interval $D \subset[0, \infty[$ and real $\varepsilon$ we consider the microcanonical Gibbs distribution

$$
\begin{aligned}
Q_{n \mid D, \varepsilon, \mathrm{per}} & \equiv Q_{n}\left(\cdot \mid N_{n} \in v_{n} D, H_{n, \mathrm{per}} \leqq v_{n} \varepsilon\right) \\
& =Q_{n}\left(\cdot \mid \rho\left(R_{n}\right) \in D, \Phi\left(R_{n}\right) \leqq \varepsilon\right)
\end{aligned}
$$

in $\Lambda_{n}$ with periodic boundary condition. As we will see, the conditioning event has positive probability for all sufficiently large $n$ whenever $\varepsilon>\inf \phi(D)$. Here $\phi:[0, \infty[\rightarrow \mathbf{R} \cup\{\infty\}$ is defined by

$$
\phi(v)=\inf \left\{\Phi(P): P \in \mathcal{P}_{\Theta}, \rho(P)=v, I(P)<\infty\right\} .
$$

Since $\Phi, \rho$ and $I$ are affine, $\phi$ is convex. $\phi$ is finite on an interval $[0, v(\varphi)[$, where $v(\varphi)=\infty$ except when $\varphi$ has a hard core, see Lemma 7.1. Intuitively, $v(\varphi)$ is the close-packing density of $\{\varphi=\infty\}$-balls.

We write $\operatorname{acc}_{n \rightarrow \infty} P^{(n)}$ for the set of all accumulation points (relative to $\tau_{\mathcal{L}}$ ) of a sequence $P^{(n)}$ in $\mathcal{P}$.

Theorem 4 Suppose $\varphi$ is superstable and regular, let $D \subset[0, \infty[$ be a nondegenerate interval with inf $D<v(\varphi)$, and $\varepsilon>\inf \phi(D)$. Then there exists some $\beta \geqq 0$ and $z>0$ such that

$$
\emptyset \neq \underset{n \rightarrow \infty}{\operatorname{acc}} Q_{n \mid D, \varepsilon, \mathrm{per}} \subset\left\{I_{z, \beta}=0\right\} \supset \underset{n \rightarrow \infty}{\operatorname{acc}} P_{n, z, \beta, \mathrm{per}} \neq \emptyset .
$$


In particular, if $\left\{I_{z, \beta}=0\right\}$ consists of a unique element $P_{z, \beta}$ then

$$
\lim _{n \rightarrow \infty} Q_{n \mid D, \varepsilon, \text { per }}=\lim _{n \rightarrow \infty} P_{n, z, \beta, \text { per }}=P_{z, \beta} .
$$

Moreover,

$$
\begin{aligned}
& \lim _{n \rightarrow \infty} v_{n}^{-1} \log Q_{n}\left(N_{n} \in v_{n} D, H_{n, \text { per }} \leqq v_{n} \varepsilon\right) \\
& \quad=-\inf \left\{I(P): P \in \mathcal{P}_{\Theta}, \rho(P) \in D, \Phi(P) \leqq \varepsilon\right\} .
\end{aligned}
$$

If $\varepsilon<\inf \phi(D)$ or $\inf D>v(\varphi)$ the limit in (2.33) equals $-\infty$.

(2.32) expresses the asymptotic equivalence of microcanonical and grandcanonical Gibbs distributions. (2.33) is an analogue of the classical existence result for the thermodynomic entropy density, cf. $[11,17]$. The difference here is that the particle number is not fixed but ranges in a whole interval, and that we use periodic boundary conditions. In addition, (2.33) provides a microcanonical Gibbs variational formula. The proof of Theorem 4 will be given in Sect. 7 which also contains some additional information on the involved functions. With the same techniques, one can derive the asymptotic equivalence of small canonical and grand canonical ensembles. In addition, an obvious extension of Lemma 7.2 in the spirit of Sects. 4 and 5 leads to a large deviation principle for the empirical fields $R_{n}$ under the microcanonical distributions $Q_{n \mid D, \varepsilon, \text { per }}$. We leave this to the reader.

\section{The energy density}

In this section we prove Theorem 1 . We begin with some basic estimates. For $P \in \mathcal{P}_{\Theta}$ we set $\rho^{(2)}(P)=P\left(N_{C}^{2}\right)$, where again $C=\Lambda_{0}$ is the centered half-open unit cube. Since

$$
N_{\Delta}^{2} \leqq m \sum_{j=1}^{m} N_{\Delta_{j}}^{2} \quad \text { when } \quad \Delta \subset \bigcup_{j=1}^{m} \Delta_{j},
$$

$\rho^{(2)}(P)<\infty$ if and only if $P \in \mathcal{P}_{\Theta}^{(2)}$, cf. (2.9).

Suppose now we are given a superstable lower regular potential $\varphi$. The presence of the purely repulsive part $\varphi^{r}$ of $\varphi$ implies that, for fixed $n, H_{n}$ tends to infinity quadratically as $N_{n} \rightarrow \infty$. This is asserted in our first lemma. Recall the definitions (2.23) and (2.10) of $T_{n}$ and $\Phi_{n}$.

Lemma 3.1 There exist constants $a>0, b<\infty$ such that for each $n \geqq 0$

$$
H_{n} \geqq a T_{n}-b N_{n}
$$

and

$$
\Phi_{n} \geqq a \rho^{(2)}-b \rho .
$$

Proof. (3.2) follows from (2.4), (2.2) and (2.3) by partitioning $A_{n}$ into $k^{d} v_{n}$ halfopen cubes $\Delta_{j}$ of size $1 / k$ (where $k$ is the smallest integer exceeding $1 / \delta$ for the number $\delta$ in (2.3)), and applying (3.1) with $\Delta=C+i, i \in L_{n}$, and $m=k^{d}$. (One can take $a=\delta / 2 k^{d} . b$ exceeds the constant in (2.2) by $\delta / 2$.) (3.3) follows from (3.2) by integration. 
As a consequence of (3.3), if $P \notin \mathcal{P}_{\Theta}^{(2)}$ then $\Phi_{n}(P)=\infty$ for all $n \geqq 0$ and thus $\lim _{n \rightarrow \infty} \Phi_{n}(P)=\infty$. To prove Theorem 1 we can therefore assume that $P \in \mathcal{P}_{\Theta}^{(2)}$. In this case, $\Phi_{n}(P)$ admits a convenient description in terms of the Palm measure $P^{\circ}$ of $P$.

Lemma 3.2 For all $P \in \mathcal{P}_{\Theta}^{(2)}$ and $n \geqq 0$ we have $\Phi_{n}(P)=P^{\circ}\left(f_{\varphi, n}\right)$, where

$$
f_{\varphi, n}(\omega)=\frac{1}{2} \sum_{0 \neq y \in \omega} \varphi(y)\left|\Lambda_{n} \cap\left(\Lambda_{n}-y\right)\right| / v_{n}, \quad \omega \in \Omega
$$

Proof. Consider the (measurable) function

$$
f_{n}(x, \omega)=\frac{1}{2} \sum_{0 \neq y \in \omega} \varphi(y) 1_{\Lambda_{n} \cap\left(A_{n}-y\right)}(x),
$$

$x \in \mathbf{R}^{d}, \omega \in \Omega$. Since $\varphi \geqq-2 b, f_{n}(x, \omega) \geqq-b 1_{\Lambda_{n}}(x) N_{n}\left(\vartheta_{-x} \omega\right)$. Also,

$$
\int P^{\circ}(d \omega) \int d x 1_{\Lambda_{n}}(x) N_{n}\left(\vartheta_{-x} \omega\right)=P\left(N_{n}^{2}\right)<\infty
$$

for all $P \in \mathcal{P}_{\Theta}^{(2)}$, by (2.8). Equation (2.8) therefore also holds for $f=f_{n}$. But for $f=f_{n}$, the left-hand side of $(2.8)$ coincides with $\Phi_{n}(P)$ and the right-hand side with $P^{\circ}\left(f_{\varphi, n}\right)$.

Since $\left|\Lambda_{n} \cap\left(\Lambda_{n}-y\right)\right| / v_{n} \rightarrow 1$ as $n \rightarrow \infty$, it is natural to expect that $P^{\circ}\left(f_{\varphi, n}\right) \rightarrow$ $P^{\circ}\left(f_{\varphi}\right)$, which will give us the first assertion of Theorem 1 . To make this rigorous we need the lower regularity of $\varphi$. Let again $L=\mathbf{Z}^{d}$ and, for each $i \in L$,

$$
\psi_{i}=\psi(d(C, C+i)),
$$

where $d(C, C+i)=(|i|-1)_{+}$is the distance of $C$ and $C+i$. Hypothesis (2.6) implies that $\sum_{i \in L} \psi_{i}<\infty$. Moreover,

$$
f_{\varphi} \geqq-\frac{1}{2} \sum_{i \in L} \psi_{i} N_{C+i}
$$

and the function on the right-hand side is $P^{\circ}$-integrable for all $P \in \mathcal{P}_{\Theta}^{(2)}$ because for all $i \in L$

$$
\begin{aligned}
P^{\circ}\left(N_{C+i}\right) & =\int P(d \omega) \sum_{x \in \omega \cap C} N_{C+i}\left(\vartheta_{x} \omega\right) \\
& \leqq P\left(N_{C} N_{A_{1}+i}\right) \leqq P\left(N_{C}^{2}\right)^{1 / 2} P\left(N_{\Lambda_{1}}^{2}\right)^{1 / 2} \\
& \leqq 3^{d} \rho^{(2)}(P) .
\end{aligned}
$$

In the last step we used (3.1). It follows that $\Phi(P) \equiv P^{\circ}\left(f_{\varphi}\right)$ is well-defined for all $P \in \mathcal{P}_{\Theta}^{(2)}$. We now compare $\Phi(P)$ with $\Phi_{n}(P)$.

Lemma 3.3 There exists a sequence $\varepsilon_{n} \rightarrow 0$ such that for all $P \in \mathcal{P}_{\Theta}^{(2)}$ and $n \geqq 0$,

$$
\Phi(P) \geqq \Phi_{n}(P)-\varepsilon_{n} \rho^{(2)}(P) .
$$


Proof. Let $P \in \mathcal{P}_{\Theta}^{(2)}$ and $n \geqq 0$ be given. Since $\left|\Lambda_{n} \cap\left(\Lambda_{n}-y\right)\right| / v_{n} \leqq 1,(2.5)$ and Lemma 3.2 imply that $\Phi(P) \geqq \Phi_{n}(P)-\varepsilon_{n}(P)$, where

$$
\varepsilon_{n}(P)=\frac{1}{2} \int P^{\circ}(d \omega) \sum_{0 \neq y \in \omega} \psi(y)\left|\Lambda_{n} \backslash\left(\Lambda_{n}-y\right)\right| / v_{n} .
$$

Distinguishing the cubes $C+i$ containing $y$, we conclude from (3.4) and (3.6) that $\varepsilon_{n}(P) \leqq \varepsilon_{n} \rho^{(2)}(P)$, where

$$
\varepsilon_{n}=\frac{1}{2} 3^{d} \sum_{i \in L} \psi_{i} \max _{y \in C+i}\left|\Lambda_{n} \backslash\left(\Lambda_{n}-y\right)\right| / v_{n}
$$

But the dominated convergence theorem shows that $\varepsilon_{n} \rightarrow 0$ as $n \rightarrow \infty$.

Proof of Theorem 1 We first show that, for all $P \in \mathcal{P}_{\Theta}, \lim _{n \rightarrow \infty} \Phi_{n}(P)$ exists and has the claimed value. The case $P \notin \mathcal{P}_{\Theta}^{(2)}$ was already discussed after Lemma 3.1. For $P \in \mathcal{P}_{\Theta}^{(2)}$, Lemma 3.2 and Fatou's lemma imply that

$$
\Phi(P)=P\left(\lim _{n \rightarrow \infty} \inf _{\varphi, n}\right) \leqq \lim _{n \rightarrow \infty} \inf _{n} \Phi_{n}(P)
$$

because the functions $f_{\varphi, n}$ are not less than the right-hand side of (3.5). Together with Lemma 3.3 , this shows that $\Phi(P)=\lim _{n \rightarrow \infty} \Phi_{n}(P)$.

To prove the lower semicontinuity of $\Phi$ we note first that each $\Phi_{n}$ is lower semicontinuous. This is because $H_{n}$ satisfies (2.2) and is thus the supremum of functions in $\mathcal{L}$. Now let $c \in \mathbf{R}$ and $\left(P_{\alpha}\right)_{\alpha \in D}$ be a net in $\{\Phi \leqq c\}$ which converges (in $\tau_{\mathcal{L}}$ ) to some $P \in \mathcal{P}_{\Theta}$. Then $\rho\left(P_{\alpha}\right) \rightarrow \rho(P)<\infty$. We thus can assume without loss that $s \equiv \sup _{\alpha} \rho\left(P_{\alpha}\right)<\infty$. In view of (3.3) and the first part of this proof, we have for all $\alpha \in D$

$$
c \geqq \Phi\left(P_{\alpha}\right) \geqq a \rho^{(2)}\left(P_{\alpha}\right)-b \rho\left(P_{\alpha}\right)
$$

and thus $\rho^{(2)}\left(P_{\alpha}\right) \leqq(c+b s) / a \equiv c^{\prime}$. Together with Lemma 3.3 this implies that

$$
\begin{aligned}
\Phi_{n}(P)-\varepsilon_{n} c^{\prime} & \leqq \lim _{\alpha \in D} \inf _{n} \Phi_{n}\left(P_{\alpha}\right)-\varepsilon_{n} c^{\prime} \\
& \leqq \lim _{\alpha \in D} \inf _{\alpha} \Phi\left(P_{\alpha}\right) \leqq c
\end{aligned}
$$

for all $n \geqq 0$. Letting $n \rightarrow \infty$ we see that $P \in\{\Phi \leqq c\}$.

We conclude this section proving the compactness of the level sets of the functionals $I_{z, \beta}$ defined in (2.14).

Lemma 3.4 For any two numbers $c_{1}, c_{2}$, the set $\left\{I+\Phi \leqq c_{1}+c_{2} \rho\right\}$ is $\tau_{\mathcal{L}}$-compact.

Proof. The set above is closed because $\rho$ is continuous and $I$ and $\Phi$ are lower semicontinuous. In fact, $I$ even has compact level sets, see Proposition 2.6 of [10]. The same is true for $I^{z}$, the relative entropy density with reference measure $Q^{z}$, the Poisson point random field with intensity $\rho\left(Q^{z}\right)=z>0$. It is easy to see that $I^{z}=I_{z, 0}=I-\rho \log z+z-1$. By (2.2), $\Phi \geqq-b \rho$. The set under consideration is therefore contained in the compact set $\left\{I^{z} \leqq c_{1}+z-1\right\}$, where $z=\exp \left(b+c_{2}\right)$. 


\section{The upper estimate}

Let $\varphi$ be superstable and regular and $F: \mathcal{P}_{\Theta} \rightarrow \mathbf{R} \cup\{\infty\}$ a measurable function such that $F \geqq-c(1+\rho)$ for some $c<\infty$. In this section we shall prove the following result.

Proposition 4.1 For bc $=$ per or free,

$$
\limsup _{n \rightarrow \infty} v_{n}^{-1} \log Q_{n}\left(\exp \left[-v_{n} F\left(R_{n}\right)-H_{n, \mathrm{bc}}\right]\right) \leqq-\inf \left[I+\Phi+F_{\mathrm{lsc}}\right] .
$$

In the case $b c=$ per, the above proposition follows directly from the results of [10] combined with those of Sect. 3. Namely, let $G=F+\Phi$. The integrand under consideration then equals $\exp \left[-v_{n} G\left(R_{n}\right)\right]$. By Theorem 1 and $(3.3), G \geqq-c^{\prime}(1+\rho)$ with $c^{\prime}=c+b$, and $G$ is clearly measurable because so is $\Phi$. Theorem 3.1 of [10] thus implies that the lim sup in Proposition 4.1 is not larger than $-\inf \left[I+G_{\mathrm{lsc}}\right]$. But $G_{\mathrm{lsc}} \geqq F_{\mathrm{lsc}}+\Phi$ because $\Phi$ is lower semicontinuous.

The proof of Proposition 4.1 for $b c=$ free is based on a comparison with the case of periodic boundary conditions. We need several lemmas. First, we use the regularity of $\varphi$ to estimate the interaction of suitably separated configurations. For each integer $k \geqq 0$ we define

$$
\delta_{k}=\frac{1}{2} \sum_{\ell \geqq k} \partial \psi(\ell) v_{\ell}
$$

where $\partial \psi(\ell)=\psi(\ell-1)-\psi(\ell) \geqq 0$ for $\ell \geqq 1, \partial \psi(0)=0$, and $\psi$ is as in (2.5) and (2.7). By (3.4),

$$
\psi_{i}=\sum_{\ell \geqq|i|} \partial \psi(\ell)
$$

for all $i \in L$. Hence

$$
\sum_{\ell \geqq 0} \partial \psi(\ell) v_{\ell}=\sum_{i \in L} \psi_{i}<\infty
$$

and thus $\delta_{k} \rightarrow 0$ as $k \rightarrow \infty$. For all $n \geqq 0, \omega \in \Omega_{n}$ and $\zeta \in \Omega$ we have

$$
\sum_{x \in \omega, y \in \zeta} \psi(y-x) \leqq \sum_{i \in L_{n}, j \in L} \psi_{i-j} N_{C+i}(\omega) N_{C+j}(\zeta)
$$

We estimate the long-distance contribution to the right-hand side of (4.3) in two cases. (The second case will be used later in Sect. 6.)

Lemma 4.2 For all $n \geqq 0, \omega \in \Omega_{n}$ and $k \geqq 0$,

$$
\begin{aligned}
& \sum_{i \in L_{n}, j \in L:|i-j| \geqq k} \psi_{i-j} \quad N_{C+i}(\omega) N_{C+j}(\zeta) \\
& \leqq\left\{\begin{array}{ccc}
\delta_{k}\left(1+2^{d}\right) T_{n}(\omega) & \text { if } \zeta=\omega^{(m)} & \text { for some } m \geqq n \\
\delta_{k}\left[T_{n}(\omega)+v_{n} t 2^{d}\right] & \text { if } \zeta \in \Omega(t) & \text { for some } t>0 .
\end{array}\right.
\end{aligned}
$$

Proof. In view of (4.2), the sum above is not larger than 


$$
\sum_{\ell \geqq k} \partial \psi(\ell) \sum_{i \in L_{n}, j \in L_{\ell}+i} N_{C+i}(\omega) N_{C+j}(\zeta) .
$$

Using the inequality $u v \leqq\left(u^{2}+v^{2}\right) / 2$ we obtain the upper bound

$$
\frac{1}{2} \sum_{\ell \geqq k} \partial \psi(\ell)\left[v_{\ell} T_{n}(\omega)+S_{n, \ell}(\zeta)\right]
$$

with

$$
S_{n, \ell}(\zeta)=\sum_{j \in L} N_{C+j}(\zeta)^{2} \text { card }\left(L_{n} \cap\left(L_{\ell}+j\right)\right) .
$$

For $\zeta=\omega^{(m)}$ with $m \geqq n$ we have

$$
\begin{aligned}
S_{n, \ell}\left(\omega^{(m)}\right) & =\sum_{j^{\prime} \in L_{n}} N_{C+j^{\prime}}(\omega)^{2} \sum_{i \in L_{n}} \operatorname{card}\left\{j \in L_{\ell}+i: j \equiv j^{\prime} \bmod 2 m+1\right\} \\
& \leqq v_{n}\left(2^{d} v_{\ell} / v_{m}\right) T_{n}(\omega) \leqq 2^{d} v_{\ell} T_{n}(\omega) .
\end{aligned}
$$

On the other hand, if $\zeta \in \Omega(t)$ then

$$
\begin{aligned}
S_{n, \ell}(\zeta) & \leqq\left(v_{n} \wedge v_{\ell}\right) T_{n+\ell}(\zeta) \\
& \leqq v_{n \wedge \ell} t v_{2(n \vee \ell)} \leqq t 2^{d} v_{n} v_{\ell}
\end{aligned}
$$

which implies the lemma in the second case.

Recall the notation $r(\varphi)$ in (2.7).

Lemma 4.3 For all $n \geqq 0$ and $k \geqq r(\varphi),\left|H_{n}-H_{n+k, \text { per }}\right| \leqq 2^{d} \delta_{k} T_{n}$ on $\Omega_{n}$.

Proof. After a comparison of (2.1) and (2.16), the lemma follows immediately from (2.5) and (2.7) together with (4.3) and Lemma 4.2.

Next we need to compare $R_{n}$ with $R_{n+k}$. For $n \geqq 0, s>0$ we define

$$
\Omega(s, n)=\left\{\omega \in \Omega: T_{n}(\omega) \leqq s v_{n}\right\} .
$$

Lemma 4.4 For all $k \geqq 1, s>0$ and $f \in \mathcal{L}$,

$$
\lim _{n \rightarrow \infty} \sup _{\omega \in \Omega(s, n+k)}\left|R_{n+k, \omega}(f)-R_{n, \omega}(f)\right|=0 .
$$

Proof. The case of bounded $f$ is trivial. We thus assume for simplicity that $|f| \leqq N_{\Delta}$ for some centered cube $\Delta \supset C$ with $f=f(\cdot \cap \Delta)$. For each $n$ we can write, setting $m=n+k$,

$$
\begin{aligned}
\left|R_{m, \omega}(f)-R_{n, \omega}(f)\right| \leqq & \left(v_{n}^{-1}-v_{m}^{-1}\right) \int_{\Lambda_{m}} N_{\Delta+x}\left(\omega^{(m)}\right) d x \\
& +v_{n}^{-1} \int_{\Lambda_{m} \backslash \Lambda_{n}} N_{\Delta+x}\left(\omega^{(m)}\right) d x \\
& +v_{n}^{-1} \int_{\partial \Lambda_{n}}\left(N_{\Delta+x}\left(\omega^{(m)}\right)+N_{\Delta+x}\left(\omega^{(n)}\right)\right) d x,
\end{aligned}
$$

where $\partial \Lambda_{n}=\left\{x \in A_{n}: x+\Delta \not \subset A_{n}\right\}$. On the other hand, for each $m$ with $\Lambda_{m} \supset$ $\Delta+C$ and all $V \subset \Lambda_{m}$ we have 


$$
\begin{aligned}
& \int_{V N_{\Delta+x}\left(\omega^{(m)}\right) d x} \leqq \sum_{i \in L} N_{C+i}\left(\omega^{(m)}\right)|\{x \in V:(\Delta+x) \cap(C+i) \neq \emptyset\}| \\
& \leqq 2^{d}|\Delta| \sum_{i \in L \cap(V+\Delta+C)} N_{C+i}\left(\omega^{(m)}\right) \\
& \leqq 4^{d}|\Delta| \sum_{j \in L_{m}(V)} N_{C+j}(\omega) \\
& \leqq 4^{d}|\Delta| \operatorname{card} L_{m}(V)^{1 / 2} T_{m}(\omega)^{1 / 2}
\end{aligned}
$$

with $L_{m}(V)=\left\{j \in L_{m}: j \equiv i \bmod 2 m+1\right.$ for some $\left.i \in V+\Delta+C\right\}$. In the cases $V=A_{m}, V=\Lambda_{m} \backslash A_{n}$, and $V=\partial \Lambda_{n}$ we have card $L_{m}(V) \leqq c_{A}|V|$ for some constant $c_{\Delta}<\infty$. Combining all estimates above one can now easily complete the proof.

Lemma 4.5 For any three constants $c_{1}, c_{2}, c_{3}>0$ there exists some $s=s\left(c_{1}, c_{2}, c_{3}\right)$ $<\infty$ such that

$$
Q_{n}\left(e^{c_{1} N_{n}-c_{2} T_{n}} ; \Omega(s, n)^{c}\right) \leqq e^{-c_{3} v_{n}}
$$

for all $n \geqq 0$.

Proof. Let $s$ be so large that $s c_{2} \geqq c_{3}+e^{c_{1}}-1$. The result then follows from the inequality

$$
1_{\Omega(s, n)^{c}} \leqq \exp \left[c_{2}\left(T_{n}-s v_{n}\right)\right]
$$

and the fact that $Q_{n}\left(e^{c_{1} N_{n}}\right)=\exp \left[\left(e^{c_{1}}-1\right) v_{n}\right]$.

Before completing the proof of Proposition 4.1 we need one further notation. We let $\mathcal{U}$ denote the system of all sets of the form

$$
U=\left\{\left(P_{1}, P_{2}\right) \in \mathcal{P}_{\Theta} \times \mathcal{P}_{\Theta}: \max _{1 \leqq i \leqq k}\left|P_{1}\left(f_{i}\right)-P_{2}\left(f_{i}\right)\right| \leqq \varepsilon\right\}
$$

with $k \geqq 1, \varepsilon>0$, and $f_{1}, \ldots, f_{k} \in \mathcal{L}$. By definition, $\mathcal{U}$ is a uniformity base for $\tau_{\mathcal{L}}$. Proof of Proposition 4.1 in the case $b c=$ free. For given $s>0$ and arbitrary $n$ we can write

$$
Q_{n}\left(\exp \left[-v_{n} F\left(R_{n}\right)-H_{n}\right]\right)=p_{n}(s)+q_{n}(s),
$$

where $p_{n}(s)$ is defined by restricting the integral on the left-hand side to the set $\Omega(s, n)$ and $q_{n}(s)$ is the remaining contribution corresponding to $\Omega(s, n)^{c}$. Let $\tau>0$ be any given number, and let $s=s(c+b, a, \tau+c)$ be chosen according to Lemma 4.5. Here $c$ is the constant appearing in the hypothesis $F \geqq-c(1+\rho)$, and $a, b$ are as in (3.2). It then follows that $q_{n}(s) \leqq \exp \left[-\tau v_{n}\right]$ for all $n$.

To estimate the main term $p_{n}(s)$ we choose an arbitrary integer $k \geqq r(\varphi)$. Then for each $n \geqq 0$ we can write, setting again $m=n+k$ and using Lemma 4.3 and (2.16),

$$
\begin{aligned}
p_{n}(s) & =e^{v_{m}-v_{n}} Q_{m}\left(\exp \left[-v_{n} F\left(R_{n}\right)-H_{n}\right] ; \Omega(s, n) \cap\left\{N_{\Lambda_{m} \backslash \Lambda_{n}}=0\right\}\right) \\
& \leqq \exp \left[v_{m}-v_{n}+2^{d} \delta_{k} s v_{n}\right] Q_{m}\left(\exp \left[-G_{m}\right]\right),
\end{aligned}
$$

where $G_{m}=v_{n} F\left(R_{n}\right)+v_{m} \Phi\left(R_{m}\right)+\infty \cdot 1_{\Omega(s, m)^{c}}$. Next we choose any $U \in \mathcal{U}$ and $\ell>0$ and define $F^{\ell}=F \wedge \ell$,

$$
F_{U}^{\ell}(P)=\inf \left\{F^{\ell}\left(P^{\prime}\right):\left(P, P^{\prime}\right) \in U\right\},
$$


and $G_{U}^{\ell}=F_{U}^{\ell}+\Phi$. Lemma 4.4 asserts that $\left(R_{m}, R_{n}\right) \in U$ on $\Omega(s, m)$ for sufficiently large $m$. Since $v_{n} F \geqq v_{m} F^{\ell}-\left(v_{m}-v_{n}\right) \ell$, we conclude that for these $m$

$$
v_{m}^{-1} G_{m} \geqq G_{U}^{\ell}\left(R_{m}\right)-\left(1-v_{n} / v_{m}\right) \ell .
$$

But this is all what is needed for the large deviation upper bound (3.2) of [10], cf. the proof of Lemma 5.6 there. (Measurability of $G_{U}^{\ell}$ is not required.) Hence

$$
\limsup _{n \rightarrow \infty} v_{m}^{-1} \log Q_{m}\left(\exp \left[-G_{m}\right]\right) \leqq-\inf \left[I+\left(G_{U}^{\ell}\right)_{l \mathrm{sc}}\right]
$$

In view of the lower semicontinuity of $\Phi,\left(G_{U}^{\ell}\right)_{\mathrm{lsc}} \geqq-\left(F_{U}^{\ell}\right)_{\mathrm{isc}}+\Phi$. Also, Lemma 3.4 shows that the argument of Remark 1.4 of [8] can be applied, yielding

$$
\sup _{\ell>0, U \in \mathcal{U}} \inf \left[I+\Phi+\left(F_{U}^{\ell}\right)_{1 \mathrm{sc}}\right]=\inf \left[I+\Phi+F_{\mathrm{lsc}}\right] \equiv \gamma
$$

Hence

$$
\lim _{n \rightarrow \infty} \sup _{n} v_{n}^{-1} \log p_{n}(s) \leqq 2^{d} \delta_{k} s-\gamma .
$$

Since $k$ is arbitrary, we finally get

$$
\lim _{n \rightarrow \infty} \sup _{n}^{-1} \log \left[p_{n}(s)+q_{n}(s)\right] \leqq-\gamma \wedge \tau,
$$

and letting $\tau \rightarrow \infty$ we obtain the result.

\section{The lower estimate}

We still assume that $\varphi$ is superstable and regular. Our proof of the lower bound (2.21) follows the standard device of changing the measure so that untypical events become typical, and controlling the Radon-Nikodym density by means of McMillan's theorem. But some refinements are necessary. The basic observation is that the familiar approximation of invariant by ergodic probability measures can be sharpened as follows. For $q>0$ we define

$$
\Gamma_{q}=\{\omega \in \Omega: \varphi(x-y) \leqq q \text { and }|x-y| \geqq 1 / q \text { for any two distinct } x, y \in \omega\} .
$$

It is easy to check that $\Gamma_{q}$ is measurable.

Lemma 5.1 Let $P \in \mathcal{P}_{\Theta}$ be such that $I(P)+\Phi(P)<\infty$. For each open neighbourhood $U$ of $P$ and any $\varepsilon>0$ there exists some $\Theta$-ergodic $P^{\prime} \in U$ such that $I\left(P^{\prime}\right)<I(P)+\varepsilon, \Phi\left(P^{\prime}\right)<\Phi(P)+\varepsilon$, and $P^{\prime}\left(\Gamma_{q}\right)=1$ for some $0<q<\infty$.

Proof. 1) Let $n \geqq 0$ be given. Since $\Phi(P)<\infty$, we have $\rho^{(2)}(P)<\infty$ and thus, by Lemma 3.3, $\Phi_{n}(P)<\infty$. Hence $P_{n}\left(\Gamma_{q}\right) \uparrow 1$ as $q \uparrow \infty$. Therefore we can choose a number $0<q(n)<\infty$ such that $p_{n} \equiv P_{n}\left(\Gamma_{q(n)}\right) \rightarrow 1$ as $n \rightarrow \infty$. We also fix an integer $k \geqq 1$ such that $2 k \geqq r(\varphi)$, where $r(\varphi)$ is as in (2.7). We can assume without loss that $q(n) \geqq \psi(k)$ for all $n$. In the following we use again the abbreviation $m=n+k$.

2) Let $\hat{P}^{(n)} \in \mathcal{P}$ be the probability measure relative to which the particle configurations in the disjoint blocks $A_{m}+(2 m+1) i, i \in L$, are independent with 
identical distribution $P_{n}^{\prime} \equiv P_{n}\left(\cdot \mid \Gamma_{q(n)}\right)$. This means in particular that the corridors $\left(\Lambda_{m} \backslash \Lambda_{n}\right)+(2 m+1) i, i \in L$, contain no particles. We also set

$$
P^{(n)}=v_{m}^{-1} \int_{\Lambda_{m}} \hat{P}^{(n)} \circ \vartheta_{x}^{-1} d x .
$$

It is then obvious that $P^{(n)} \in \mathcal{P}_{\Theta}$, and a standard argument shows that $P^{(n)}$ is ergodic; see, for example, Theorem 14.12 of [7]. Since $\Gamma_{q(n)}$ is $\Theta$-invariant and $\hat{P}^{(n)}\left(\Gamma_{q(n)}\right)=$ 1 , it also follows that $P^{(n)}\left(\Gamma_{q(n)}\right)=1$.

3) Next we show that $\lim _{\sup _{n \rightarrow \infty}} I\left(P^{(n)}\right) \leqq I(P)$. By an analogue of Lemma 5.5 of [10],

$$
\begin{aligned}
I\left(P^{(n)}\right) & \leqq I\left(P_{n}^{\prime} ; Q_{m}\right) / v_{m} \\
& =\left[I\left(P_{n}\left(\cdot \mid \Gamma_{q(n)}\right) ; Q_{n}\right)+v_{m}-v_{n}\right] / v_{m} .
\end{aligned}
$$

On the other hand, since $I(P)<\infty$ we have $P_{n} \ll Q_{n}$ with a density $f_{n}$, and thus

$$
\begin{aligned}
I\left(P_{n}\left(\cdot \mid \Gamma_{q(n)}\right) ; Q_{n}\right) & =P_{n}\left(\log f_{n} / p_{n} ; \Gamma_{q(n)}\right) / p_{n} \\
& \leqq\left(I\left(P_{n} ; Q_{n}\right)+1\right) / p_{n}-\log p_{n}
\end{aligned}
$$

because $x \log x \geqq-1$ for all $x \geqq 0$ and thus

$$
P_{n}\left(\log f_{n} ; \Gamma_{q(n)}^{c}\right)=Q_{n}\left(f_{n} \log f_{n} ; \Gamma_{q(n)}^{c}\right) \geqq-1 .
$$

Since $\left(v_{m}-v_{n}\right) / v_{m} \rightarrow 0$ and $p_{n} \rightarrow 1$ as $n \rightarrow \infty$, the desired result follows.

4) The regularity of $\varphi$ implies that $\lim \sup _{n \rightarrow \infty} \Phi\left(P^{(n)}\right) \leqq \Phi(P)$. Indeed, since obviously $P^{(n)} \in \mathcal{P}_{\Theta}^{(2)},(2.11)$ and (2.8) yield

$$
\begin{aligned}
\Phi\left(P^{(n)}\right) & =\int P^{(n)}(d \omega) \sum_{x \in \omega \cap C} f_{\varphi}\left(\vartheta_{x} \omega\right) \\
& =v_{m}^{-1} \int_{\Lambda_{m}} d u \int \hat{P}^{(n)}(d \omega) \frac{1}{2} \sum_{x, y \in \omega, y \neq x} 1_{C+u}(x) \varphi(y-x) \\
& =v_{m}^{-1} \int \hat{P}^{(n)}(d \omega) \frac{1}{2} \sum_{x, y \in \omega, y \neq x} \varphi(y-x)\left|\Lambda_{m} \cap(x-C)\right| \\
& =a_{n}+b_{n} .
\end{aligned}
$$

Here $a_{n}=v_{m}^{-1} \hat{P}^{(n)}\left(H_{n}\right)$ and

$$
b_{n}=v_{m}^{-1} \int \hat{P}^{(n)}(d \omega) \frac{1}{2} \sum_{x \in \omega \cap A_{n}, y \in \omega \backslash \Lambda_{m}} \varphi(y-x),
$$

and we have used that for $\hat{P}^{(n)}$-almost all $\omega$ and all $x \in \omega$ either $x \in \Lambda_{n}$, and thus $x-C \subset A_{m}$, or $A_{m} \cap(x-C)=\emptyset$. Now,

$$
\begin{aligned}
a_{n} & =P_{n}\left(H_{n} ; \Gamma_{q(n)}\right) / p_{n} v_{m} \\
& \leqq \Phi_{n}(P) v_{n} / p_{n} v_{m}+b P\left(N_{n} ; \Gamma_{q(n)}^{c}\right) / p_{n} v_{m} \\
& \leqq \Phi_{n}(P)(1+o(1))+b P\left(N_{n}^{2}\right)^{1 / 2}\left(1-p_{n}\right)^{1 / 2} / p_{n} v_{m} \\
& =\Phi_{n}(P)(1+o(1))+o(1)
\end{aligned}
$$


because of (2.2) and (3.1), and $b_{n}=o(1)$ because of (2.7), (4.3), the inequality

$$
\hat{P}^{(n)}\left(N_{C+i} N_{C+j}\right)=\hat{P}^{(n)}\left(N_{C+i}\right) \hat{P}^{(n)}\left(N_{C+j}\right) \leqq \rho(P)^{2} / p_{n}^{2}
$$

for $i \in L_{n}, j \notin L_{m}$, and the summability of $i \rightarrow \psi_{i}$.

5) We finally show that $P^{(n)}(f) \rightarrow P(f)$ as $n \rightarrow \infty$ for all $f \in \mathcal{L}$. Since this is quite obvious when $f$ is bounded, we assume for simplicity that $|f| \leqq N_{\Delta}$, where $\Delta$ is any cube with $f=f(\cdot \cap \mathcal{A})$. Then we can write

$$
\left|P^{(n)}(f)-P(f)\right| \leqq\left(s_{n}+t_{n}\right) / v_{m}
$$

with

$$
s_{n}=\int_{\left\{x+\Delta \subset \Lambda_{m}\right\}} d x\left|P_{n}\left(f \circ \vartheta_{x} \mid \Gamma_{q(n)}\right)-P\left(f \circ \vartheta_{x}\right)\right|
$$

and

$$
t_{n}=\int_{\partial \Lambda_{m}} d x\left[\hat{P}^{(n)}\left(N_{\Delta+x}\right)+P\left(N_{\Delta+x}\right)\right],
$$

where $\partial \Lambda_{m}$ is as in the proof of Lemma 4.4. Now $P\left(N_{\Delta+x}\right)=|\Delta| \rho(P)$,

$$
\hat{P}^{(n)}\left(N_{\Delta+x}\right)=P_{n}\left(N_{\Delta+x \bmod 2 m+1} \mid \Gamma_{q(n)}\right) \leqq|\Delta| \rho(P) / p_{n},
$$

and therefore $t_{n}=o\left(v_{m}\right)$. On the other hand, the integrand defining $s_{n}$ is at most

$$
\begin{aligned}
& \left|1-p_{n}^{-1}\right| P_{n}\left(N_{\Delta+x} ; \Gamma_{q(n)}\right)+P_{n}\left(N_{\Delta+x} ; \Gamma_{q(n)}^{c}\right) \\
& \quad \leqq|\Delta| \rho(P)\left|1-p_{n}^{-1}\right|+P\left(N_{\Delta}^{2}\right)^{1 / 2}\left(1-p_{n}\right)^{1 / 2}
\end{aligned}
$$

whence $s_{n}=o\left(v_{m}\right)$. This completes the last step of the proof.

The main advantage of the classes

$$
\mathcal{P}_{\Theta, q}=\left\{P \in \mathcal{P}_{\Theta}: P\left(\Gamma_{q}\right)=1\right\}
$$

is the following continuity property of $\Phi$.

Lemma 5.2 For each $q>0, \Phi$ is continuous on $\mathcal{P}_{\Theta, q}$.

Proof. For each $n \geqq 0, H_{n}$ is local and bounded on $\Gamma_{q}$. Hence $\Phi_{n}$ is continuous on $\mathcal{P}_{\Theta, q}$. Also, a glance at Lemma 3.3 and its proof shows that the convergence $\Phi_{n} \rightarrow \Phi$ is uniform on $\mathcal{P}_{\Theta, q}$. This gives the result.

Let $r \geqq r(\varphi)$ be a fixed integer. For $n \geqq r$ we define the modified empirical fields

$$
R_{n, \omega}^{\#}=R_{n, \omega \cap A_{n-r}} .
$$

Lemma 5.3 For each ergodic $P \in \mathcal{P}_{\Theta}, R_{n}^{\#} \rightarrow P$ in $P$-probability as $n \rightarrow \infty$.

Proof. This follows in the same way as (1.2); cf. the proof of Remark 2.4 in [10].

Let $F$ be as in Theorem 2 .

Proposition 5.4 For all $P \in \mathcal{P}_{\Theta}$ and bc $\in\{$ per, free $\}$,

$$
\liminf _{n \rightarrow \infty} v_{n}^{-\mathrm{l}} \log Q_{n}\left(\exp \left[-v_{n} F\left(R_{n}\right)-H_{n, \mathrm{bc}}\right]\right) \geqq-\left[I(P)+\Phi(P)+F^{\mathrm{usc}}(P)\right] .
$$


Proof. We may assume without loss that the right-hand side of the asserted inequality, denoted by $-\gamma_{P}$, is finite. By Lemma 5.1 , we can even assume that $P$ is ergodic and supported on $\Gamma=\Gamma_{q}$ for some $q \geqq \psi(r)$. For $n \geqq r$ let $P_{n}^{\#}=P_{n-r}$. We think of $P_{n}^{\#}$ as a measure on $\Omega_{n}$ which leaves $D_{n}=A_{n} \backslash \Lambda_{n-r}$ free of particles. Since $I(P)<\infty, P_{n-r} \ll Q_{n-r}$ with a density $f_{n-r}$. Hence $P_{n}^{\#} \ll Q_{n}$ with the density $f_{n}^{\#}=1_{\left\{N_{D_{n}}=0\right\}} f_{n-r} \exp \left[v_{n}-v_{n-r}\right]$. Given any $\varepsilon>0$, we define

$$
A_{n}=\left\{F\left(R_{n}\right)<F^{\mathrm{usc}}(P)+\varepsilon, v_{n}^{-1} H_{n, \mathrm{bc}}<\Phi(P)+\varepsilon, v_{n}^{-1} \log f_{n}^{\#}<I(P)+\varepsilon\right\} .
$$

A well-known estimate (see, e.g.,[10]) then shows that the lim inf in the proposition is not less than

$$
-\gamma_{P}-3 \varepsilon+\liminf _{n \rightarrow \infty} v_{n}^{-1} \log P_{n}^{\#}\left(A_{n}\right) .
$$

It is therefore sufficient to show that $P_{n}^{\#}\left(A_{n}\right) \rightarrow 1$. But

$$
P_{n}^{\#}\left(F\left(R_{n}\right)<F^{\mathrm{usc}}(P)+\varepsilon\right)=P\left(F\left(R_{n}^{\#}\right)<F^{\mathrm{usc}}(P)+\varepsilon\right) \rightarrow 1
$$

because of Lemma 5.3, and

$$
P_{n}^{\#}\left(v_{n}^{-1} \log f_{n}^{\#}<I(P)+\varepsilon\right)=P_{n-r}\left(v_{n}^{-1} \log f_{n-r}+1-v_{n}^{-1} v_{n-r}<I(P)+\varepsilon\right) \rightarrow 1
$$

by McMillan's theorem $[5,14]$. Moreover, for bc $=$ per we obtain from Lemmas 5.2 and 5.3 that

$$
P_{n}^{\#}\left(v_{n}^{-1} H_{n, \text { per }}<\Phi(P)+\varepsilon\right)=P\left(\Phi\left(R_{n}^{\#}\right)<\Phi(P)+\varepsilon\right) \rightarrow 1
$$

because $P\left(R_{n}^{\#} \in \mathcal{P}_{\Theta, q}\right)=1$. Thus $P_{n}^{\#}\left(A_{n}\right) \rightarrow 1$ for $\mathrm{bc}=$ per, and the same result follows for $\mathrm{bc}=$ free because

$$
\sup _{\omega \in \Gamma}\left|H_{n}\left(\omega \cap A_{n-r}\right)-H_{n, \text { per }}\left(\omega \cap A_{n-r}\right)\right| \leqq \frac{1}{2} v^{2} \sum_{i \in L_{n-r, j} \notin L_{n}} \psi_{i-j}=o\left(v_{n}\right),
$$

where $v=\sup N_{C}\left(\Gamma_{q}\right)$. This proves the proposition.

The proof of Theorem 2 is now completed as follows. First, there is no loss in assuming $\beta=1$. Next, we apply Propositions 4.1 and 5.4 to $F_{z}=-\rho \log z$. Since $\rho\left(R_{n}\right)=N_{n} / v_{n}$, this proves (2.22). Combining (2.22) with Proposition 4.1 (applied to $F+F_{z}$ instead of $F$ ) we arrive at the upper bound (2.20), and the lower bound (2.21) follows in the same way from (2.22) and Proposition 5.4.

\section{Uniform estimates for tempered boundary conditions}

This section is devoted to the proof of Theorem 3. We thus assume that $\varphi$ is regular and non-integrably divergent at the origin. By Proposition 3.2.8 of [17], the stable part $\varphi^{s}$ of $\varphi$ may be chosen bounded. So we can assume that the repulsive part $\varphi^{r}$ satisfies (2.26). This gives us the following sharpening of the basic inequality (3.2).

Lemma 6.1 There exists a constant $b<\infty$ and an increasing function $h: \mathbf{Z}_{+} \rightarrow$ $\left[0, \infty\left[\right.\right.$ such that $h(0)=0, h(\ell) / \ell^{2} \rightarrow \infty$ as $\ell \rightarrow \infty$, and for all $n \geqq 0$

$$
H_{n} \geqq T_{n}^{h}-b N_{n},
$$


where

$$
T_{n}^{h}=\sum_{i \in L_{n}} h\left(N_{C+i}\right)
$$

Proof. This is essentially Lemma 1 of [3]. For completeness we sketch the argument. Since the stable part of $\varphi$ satisfies (2.2), and in view of the remarks above, we can assume without loss that $\varphi=\varphi^{r} \geqq 0$. We can further assume that $\chi(1)=0$ for the function $\chi$ satisfying (2.26) and (2.27). It is also sufficient to prove (6.1) for $n=1$.

Let $\omega \subset C$ be a configuration of some cardinality $N \geqq 3^{d}$ and $K \geqq 3$ the integer part of $N^{1 / d}$. Using (2.26) and writing $\chi_{k}=\chi(1 / k)$ we then have

$$
\varphi(x) \geqq \sum_{k=2}^{K}\left(\chi_{k}-\chi_{k-1}\right) 1_{\{|x| \leqq 1 / k\}}
$$

for all $x$. For each $k \geqq 2$ we divide $C$ into $k^{d}$ cubes $V(i)$ of size $1 / k$, and we let $N_{i}=$ card $\omega \cap V(i), i=1, \ldots, k^{d}$. Then

$$
\begin{aligned}
\frac{1}{2} \sum_{x, y \in \omega, x \neq y} 1_{\{|x-y| \leqq 1 / k\}} & \geqq \sum_{i=1}^{k^{d}}\left(\begin{array}{c}
N_{i} \\
2
\end{array}\right) \\
& \geqq \frac{1}{2}\left(k^{-d} N^{2}-N\right) .
\end{aligned}
$$

Summing over $k$ we thus obtain

$$
\begin{aligned}
H_{1}(\omega) & \geqq \frac{1}{2} \sum_{k=2}^{K}\left(\chi_{k}-\chi_{k-1}\right)\left(k^{-d} N^{2}-N\right) \\
& =N^{2} \frac{1}{2} \sum_{k=2}^{K-1} \chi_{k}\left(k^{-d}-(k+1)^{-d}\right)+\frac{1}{2} \chi_{k}\left(K^{-d} N^{2}-N\right) \\
& \geqq N^{2} d 3^{-d} \frac{1}{2} \int_{1 /(K-1)}^{1} \chi(s) s^{d-1} d s \equiv h(N)
\end{aligned}
$$

Setting $h(N)=0$ for $N<3^{d}$ we obtain the lemma.

The next lemma establishes a simple relation between superquadratic and subquadratic functions on $\mathbf{Z}_{+}$by means of the Legendre-Fenchel transform. Observe that this transform preserves the parabola $\ell \rightarrow \ell^{2} / 2$.

Lemma 6.2 Suppose $g: \mathbf{Z}_{+} \rightarrow\left[0, \infty\left[\right.\right.$ is such that $g(0)=0$ and $g(\ell) / \ell^{2} \rightarrow \infty$ as $\ell \rightarrow \infty$. Let $g^{*}: \mathbf{Z}_{+} \rightarrow\left[0, \infty\left[\right.\right.$ be defined by $g^{*}(m)=\sup _{\ell}[m \ell-g(\ell)]$. Then $g^{*}$ is increasing, $g^{*}(0)=0$, and $g^{*}(m) / m^{2} \rightarrow 0$ as $m \rightarrow \infty$.

Proof. This is a straightforward computation. Note that no convexity of $g$ is required.

The following lemma will allow us to reduce the case of tempered boundary conditions to that of the free boundary condition.

Lemma 6.3 Let $\varepsilon>0, t>0$ be given. If $n$ is sufficiently large, 


$$
\inf _{\zeta \in \Omega(t)} H_{n, \zeta} \geqq H_{n}-\varepsilon T_{n}^{h}-\varepsilon v_{n},
$$

where $h$ is as in Lemma 6.1.

Proof. In view of $(2.25),(2.5)$ and (4.3) we must show that

$$
\sup _{\zeta \in \Omega(t)} \sum_{i \in L_{n}, j \notin L_{n}} \psi_{i-j} N_{C+i} N_{C+j}(\zeta) \leqq \varepsilon T_{n}^{h}+\varepsilon v_{n}
$$

for large $n$. Let $q$ be such that $h(\ell) \geqq \ell^{2}$ for all $\ell \geqq q$. Then $T_{n} \leqq q^{2} v_{n}+T_{n}^{h}$ for all $n$. By Lemma 4.2 , the contribution of all $i, j$ with $|i-j| \geqq k$ to the sum on the left-hand side of (6.3) is not larger than $\delta_{k}\left[T_{n}^{h}+v_{n}\left(t 2^{d}+q^{2}\right)\right]$. Choosing $k$ sufficiently large we can achieve that this is less than $\varepsilon\left(T_{n}^{h}+v_{n}\right) / 2$.

To estimate the remaining part of the sum in (6.3) we define $g(\ell)=\ell h(\ell)^{1 / 2}$, $\ell \geqq 0 . g$ is increasing with $g(0)=0$ and satisfies $g(\ell) / \ell^{2} \rightarrow \infty$ and $g(\ell) / h(\ell) \rightarrow 0$ as $\ell \rightarrow \infty$. Let $g^{*}$ be as in Lemma 6.2. Then $\ell m \leqq g(\ell)+g^{*}(m)$ for all $\ell, m \geqq 0$ and therefore

$$
\begin{gathered}
\sum_{i \in L_{n}, j \notin L_{n}:|i-j|<k} \psi_{i-j} N_{C+i} N_{C+j}(\zeta) \\
\leqq \Psi \sum_{i \in L_{n} \backslash L_{n-k}} g\left(N_{C+i}\right)+\Psi \sum_{j \in L_{n+k} \backslash L_{n}} g^{*}\left(N_{C+j}(\zeta)\right)
\end{gathered}
$$

for all $n \geqq k$ and $\zeta \in \Omega$. Here $\Psi=\sum_{i \in L} \psi_{i}$. Since $g(\ell) / h(\ell) \rightarrow 0$ as $\ell \rightarrow \infty$, the first expression on the right-hand side of (6.4) is at most $\varepsilon T_{n}^{h} / 2+O\left(v_{n}-v_{n-k}\right)$. Similarly, Lemma 6.2 implies that the second term on the right-hand side of (6.4) admits a bound of the form

$$
\varepsilon T_{n+k}(\zeta) / 4 t+O\left(v_{n+k}-v_{n}\right) .
$$

Since $v_{n+k}-v_{n}=o\left(v_{n}\right)$, it follows that, for $\zeta \in \Omega(t)$ and sufficiently large $n$, the left-hand side of (6.4) is not larger than $\varepsilon\left(T_{n}^{h}+v_{n}\right) / 2$. This proves (6.3).

Proof of Theorem 3, assertion (a). Let $F$ and $t$ be as in the theorem, and let $0<\varepsilon<1 / 2$ be given. In view of Lemma 6.3 we have for sufficiently large $n$

$$
\begin{aligned}
\bar{p}_{n}(t) & \equiv \sup _{\zeta \in \Omega(t)} Q_{n}\left(\exp \left[-v_{n} F\left(R_{n}\right)-H_{n, \zeta]}\right)\right. \\
& \leqq e^{\varepsilon v_{n}} Q_{n}\left(\exp \left[-v_{n} F\left(R_{n}\right)-H_{n}+\varepsilon T_{n}^{h}\right]\right) .
\end{aligned}
$$

By Lemma 6.1 and the hypothesis on $F$, the exponent in the last integral is not larger than $c v_{n}+(c+b) N_{n}-(1-\varepsilon) T_{n}^{h}$. An analogue of Lemma 4.5 thus shows that we may restrict the integral to a set of the form $\left\{T_{n}^{h} \leqq s v_{n}\right\}$ with suitable $s<\infty$, the remainder being at most $e^{-\tau v_{n}}$ for any prescribed $\tau>0$. Together with Proposition 4.1 ( for $b c=$ free ), this gives the estimate

$$
\limsup _{n \rightarrow \infty} v_{n}^{-1} \log \bar{p}_{n}(t) \leqq(\varepsilon+\varepsilon s-\gamma) \vee(-\tau),
$$

where $\gamma=\inf \left[I+\Phi+F_{\text {lsc }}\right]$. Letting first $\varepsilon \rightarrow 0$ and then $\tau \rightarrow \infty$ we obtain the uniform upper bound 


$$
\limsup _{n \rightarrow \infty} v_{n}^{-1} \log \bar{p}_{n}(t) \leqq-\gamma .
$$

To obtain a lower estimate of

$$
\underline{p}_{n}(t) \equiv \inf _{\zeta \in \Omega(t)} Q_{n}\left(\exp \left[-v_{n} F\left(R_{n}\right)-H_{n, \zeta}\right]\right)
$$

we can proceed just as in the proof of Proposition 5.4, the only difference being that now we must show that

$$
\inf _{\zeta \in \Omega(t)} P_{n-r}\left(v_{n}^{-1} H_{n, \zeta}<\Phi(P)+\varepsilon\right) \rightarrow 1 \quad \text { as } \quad n \rightarrow \infty
$$

for any given $\varepsilon>0$, each ergodic $P \in \mathcal{P}_{\Theta}$ which is supported on some $\Gamma_{q}$, and suitable $r \geqq r(\varphi)$. But (2.7), (4.3) and Lemma 4.2 imply that

$$
\sup _{\zeta \in \Omega(t)} H_{n, \zeta} \leqq H_{n}+\varepsilon v_{n} / 2 \quad \text { on } \quad \Gamma_{q} \cap \Omega_{n-r}
$$

when $r$ is large enough, and we know from the proof of Proposition 5.4 that

$$
P_{n-r}\left(v_{n}^{-1} H_{n}<\Phi(P)+\varepsilon / 2\right) \rightarrow 1 \quad \text { as } n \rightarrow \infty
$$

We thus arrive at the uniform lower bound

$$
\liminf _{n \rightarrow \infty} v_{n}^{-1} \log \underline{p}_{n}(t) \geqq-\inf \left[I+\Phi+F^{\mathrm{usc}}\right]
$$

Assertion (a) of Theorem 3 now follows from (6.5) and (6.6) in the same way as Theorem 2 from Propositions 4.1 and 5.4.

We now turn to the large deviation principle for tempered Gibbs measures. Our main tool are the remarkable probability estimates of Ruelle [18]. (Similar estimates appear in [3].) The implication (a) $\Rightarrow(b)$ of his Corollary 5.3 gives us the following.

Proposition 6.4 For given $z, \beta>0$, there exist constants $\gamma, \delta>0$ such that, for all tempered Gibbs measures $P$ with parameters $z, \beta$ and all $n \geqq 0, P_{n}$ is absolutely continuous relative to $Q_{n}$ with a density $f_{n}$ satisfying $f_{n} \leqq \exp \left[v_{n}-\gamma T_{n}+\delta N_{n}\right]$.

For $t>0$ and $n \geqq 0$ we define

$$
\Omega_{n}(t)=\left\{\zeta \in \Omega: \zeta \backslash \Lambda_{n} \in \Omega(t)\right\} .
$$

Corollary 6.5 Let $z, \beta>0$ and $\tau, c>0$ be any constants. Then there exists some $t>0$ such that, for each tempered Gibbs measure $P$ with parameters $z, \beta$ and all $n \geqq 0$,

$$
P\left(e^{c N_{n}} ; \Omega_{n}(t)^{c}\right) \leqq e^{-\tau v_{n}}
$$

Proof. We may assume that $\tau>\log 2$. Let $\gamma, \delta$ be as in Proposition 6.4 and $t$ so large that $t \gamma-e^{c+\delta} \geqq \tau$. Then we can write 


$$
\begin{aligned}
P\left(e^{c N_{n}} ; \Omega_{n}(t)^{c}\right) & \leqq \sum_{\ell>n} P\left(e^{c N_{n}} ; T_{\ell}>t v_{\ell}\right) \\
& \leqq \sum_{\ell>n} P\left(\exp \left[c N_{\ell}+\gamma T_{\ell}-\gamma t v_{\ell}\right]\right) \\
& \leqq \sum_{\ell>n} Q\left(\exp \left[v_{\ell}(c+\delta) N_{\ell}-\gamma t v_{\ell}\right]\right) \\
& \leqq \sum_{\ell>n} \exp \left[-\tau v_{\ell}\right] .
\end{aligned}
$$

This implies the corollary because $v_{\ell}>v_{n}+\ell-n$ when $\ell>n$.

Proof of Theorem 3, assertion (b). Let $z, \beta>0$ and $P$ be a tempered Gibbs measure for $z, \beta$. Also, let $F: \mathcal{P}_{\Theta} \rightarrow \mathbf{R} \cup\{\infty\}$ be such that $F \geqq-c(1+\rho)$ for some $c<\infty, \tau>c$ an arbitrary constant, and $t$ as in Corollary 6.5. Then

$$
P\left(e^{v_{n} F\left(R_{n}\right)} ; \Omega_{n}(t)^{c}\right) \leqq e^{-(\tau-c) v_{n}}
$$

and

$$
\begin{aligned}
P\left(e^{v_{n} F\left(R_{n}\right)} ; \Omega_{n}(t)\right) & =\int_{\Omega_{n}(t)} P(d \zeta) P_{n, z, \beta, \zeta}\left(e^{v_{n} F\left(R_{n}\right)}\right) \\
& \leqq \sup _{\zeta \in \Omega(t)} P_{n, z, \beta, \zeta}\left(e^{v_{n} F\left(R_{n}\right)}\right)
\end{aligned}
$$

for all $n$. The uniform upper bound (2.28) thus implies

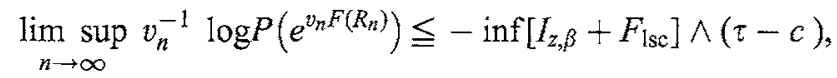

and letting $\tau \rightarrow \infty$ we obtain the upper large deviation bound for $P$. The lower bound follows from the inequality

$$
P\left(e^{v_{n} F\left(R_{n}\right)}\right) \geqq P(\Omega(t)) \inf _{\zeta \in \Omega(t)} P_{n, z, \beta, \zeta}\left(e^{v_{n} F\left(R_{n}\right)}\right)
$$

together with (2.29) and the fact that $P(\Omega(t))>0$ for sufficiently large $t$.

\section{The equivalence of ensembles}

In this section we prove Theorem 4. let $\varphi$ be superstable and regular. We first look at the function $\phi$ defined in (2.31). Let

$$
\Omega_{\varphi}=\{\omega \in \Omega: \varphi(x-y)<\infty \text { for all } x, y \in \omega, x \neq y\}
$$

and

$$
\nu(\varphi)=\sup \left\{\rho(P): P \in \mathcal{P}_{\Theta}, I(P)<\infty, P\left(\Omega_{\varphi}\right)=1\right\} .
$$

Clearly, $v(\varphi)$ depends on $\varphi$ only via the set $\{\varphi=\infty\}$. If $\varphi$ is finite (except possibly at the origin) then $v(\varphi)=\infty$. For in this case we have $\Omega_{\varphi}=\Omega$ so that the Poisson point random fields $P=Q^{z}$ of arbitrarily large intensity $z$ appear on the right-hand side of (7.1).

Lemma $7.1 \phi$ is convex. On $\left[0, v(\varphi)\left[, \phi\right.\right.$ is finite and continuous. Also, $\phi(v) \geqq a v^{2}-$ $b v$ for all $v \geqq 0$ and the constants $a, b$ in (3.3), and if $\lambda \equiv \int \varphi(x) d x$ is finite then $\phi(v) \leqq \lambda v^{2} / 2$ for all $v \geqq 0$. (In particular, it follows that $\lambda \geqq a$.) 
Proof. The inequalities for $\phi$ follow from (3.3) and the easily verified fact that $\Phi\left(Q^{z}\right)=\lambda z^{2} / 2$ for all $z>0$. It only remains to show that $\phi$ is finite on $[0, v(\varphi)[$. For, together with the obvious convexity of $\phi$, this will imply that $\phi$ is continuous on $] 0, v(\varphi)[$. The continuity of $\phi$ at 0 is clear because $\phi(0)=0$ and $\phi(v) \geqq-b v$.

To prove the finiteness of $\phi$ we fix any $v<v(\varphi)$. By (7.1) there exists some $P \in \mathcal{P}_{\Theta}$ such that $\rho(P)>v, I(P)<\infty$, and $P\left(\Omega_{\varphi}\right)=1$. A glance at the proof of Lemma 5.1 shows that its hypothesis $\Phi(P)<\infty$ can be replaced by the condition $P\left(\Omega_{\varphi}\right)=1$. Therefore we can find some $P^{\prime} \in \mathcal{P}_{\Theta}$ such that $\rho\left(P^{\prime}\right)>v, I\left(P^{\prime}\right)<\infty$, and $P^{\prime}\left(\Gamma_{q}\right)=1$ for some $q<\infty$. Since $\Gamma_{q}$ is $\Theta$-invariant, the Palm measure of $P^{\prime}$ is also supported on $\Gamma_{q}$. This and the regularity of $\varphi$ immediately imply that $\Phi\left(P^{\prime}\right)<\infty$. Writing $s=v / \rho\left(P^{\prime}\right)$ we thus obtain that $P^{\prime \prime} \equiv s P^{\prime}+(1-s) \delta_{\emptyset}$ has the properties $\rho\left(P^{\prime \prime}\right)=v, \Phi\left(P^{\prime \prime}\right)<\infty$, and $I\left(P^{\prime \prime}\right)<\infty$. Hence $\phi(v)<\infty$.

In view of (7.3) and (7.7) below, the function $\phi(v)$ coincides with the function $\varepsilon_{0}(\rho)$ on page 50 of [17]. Note, however, that not necessarily $\phi(v) \rightarrow \infty$ when $v \rightarrow v(\varphi)<\infty$; indeed, if $\varphi$ is a pure hard core potential (taking only the values 0 and $\infty)$ then $\phi(v)=0$ for all $v<v(\varphi)$.

Next we define

$$
s(v, \varepsilon)=-\inf \left\{I(P): P \in \mathcal{P}_{\Theta}, \rho(P)=v, \Phi(P) \leqq \varepsilon\right\} .
$$

Clearly, $s(\cdot, \cdot)$ is increasing in $\varepsilon$ and concave, and (2.31) means that

$$
\phi(v)=\inf \{s(v, \cdot)>-\infty\} \quad \text { for all } \quad v \geqq 0 .
$$

Using the lower semicontinuity of $\Phi$ and the fact that $I$ has compact level sets [10] we also see that $s(\cdot, \cdot)$ is upper semicontinuous. $s(\cdot, \cdot)$ is the entropy density, as is shown in the next lemma which proves (2.33).

Lemma 7.2 Let $D \subset[0, \infty[$ be a nondegenerate interval with inf $D<\nu(\varphi)$, and let $\varepsilon>\inf \phi(D)$. Then

$$
\lim _{n \rightarrow \infty} v_{n}^{-1} \log Q_{n}\left(N_{n} \in v_{n} D, H_{n, \mathrm{per}} \leqq v_{n} \varepsilon\right)=s(D, \varepsilon) \equiv \sup _{v \in D} s(v, \varepsilon)
$$

If $\inf D>v(\varphi)$ or $\varepsilon<\inf \phi(D)$ then the limit above equals $-\infty$.

Proof. Let $\bar{D}$ be the closure and $D^{o}$ the interior of $D$. By the continuity of $\phi$ on $\left[0, v(\varphi)\left[, \inf \phi(\bar{D})=\inf \phi\left(D^{o}\right)=\inf \phi(D)\right.\right.$. In view of the continuity of $\rho$ and the lower semicontinuity of $\Phi$, the set $A=\{\rho \in \bar{D}, \Phi \leqq \varepsilon\}$ is closed. The upper bound (2.20) for $\beta=0, z=1$ thus implies that

$$
\limsup _{n \rightarrow \infty} v_{n}^{-1} \log Q_{n}\left(R_{n} \in A\right) \leqq-\min I(A)
$$

( $I$ attains its minimum over $A$ because $I$ has compact level sets.) On the other hand, consider the convex set $U=\left\{\rho \in D^{o}, \Phi<\varepsilon\right\}$. Since $\varepsilon>\inf \phi\left(D^{o}\right), U \cap\{I<$ $\infty\} \neq \emptyset$. A standard convexity argument (together with the fact that $I, \rho$ and $\Phi$ are affine) thus shows that inf $I(U)=\inf I(A)=-s(D, \varepsilon)$. But

$$
\liminf _{n \rightarrow \infty} v_{n}^{-1} \log Q_{n}\left(R_{n} \in U\right) \geqq-\inf I(U)
$$

by the arguments of Sect. 5 . Indeed, let $P \in U$ be such that $I(P)<\infty$. For given $\varepsilon>0$, Lemma 5.1 provides us with some ergodic $P^{\prime} \in U$ such that $I\left(P^{\prime}\right)<I(P)+$ 
$\varepsilon$ and $P^{\prime}\left(\Gamma_{q}\right)=1$ for some $q>0$. As in the proof of Proposition 5.4, we have the estimate

$$
v_{n}^{-1} \log Q_{n}\left(R_{n} \in U\right) \geqq-I\left(P^{\prime}\right)-\varepsilon+v_{n}^{-1} \log P^{\prime}\left(R_{n}^{\#} \in U, v_{n}^{-1} \log f_{n}^{\#} \leqq I\left(P^{\prime}\right)+\varepsilon\right),
$$

and McMillan's theorem $[5,14]$ and Lemmas 5.2 and 5.3 show that the last probability tends to 1 as $n \rightarrow \infty$. The second statement of the lemma follows from (7.3) and (7.4).

Proposition 7.3 Suppose $D \subset[0, \infty[$ is a nondegenerate interval with $\inf D<v(\varphi)$, and let $\varepsilon>\inf \phi(D)$. Then the sequence $\left(Q_{n \mid D, \varepsilon, \mathrm{per}}\right)_{n \geqq 0}$ is relatively compact (in $\left.\tau_{\mathcal{L}}\right)$, and every accumulation point belongs to the set $M_{D, \varepsilon}$ of all I-minimizers in $\{\rho \in \bar{D}, \Phi \leqq \varepsilon\}$.

Proof. Lemma 7.2 implies that the conditioning event in (2.30) has positive probability for sufficiently large $n$. Thus, for all these $n, Q_{n \mid D, \text {, per }}$ is well-defined. Let $\hat{P}^{(n)} \in \mathcal{P}$ denote the measure relative to which the configurations in the disjoint blocks $A_{n}+(2 n+1) i, i \in L$, are independent with identical distribution $Q_{n \mid D, \varepsilon, \mathrm{per}}$, and $P^{(n)} \in \mathcal{P}_{\Theta}$ the invariant average of $\hat{P}^{(n)} \circ \vartheta_{x}^{-1}, x \in \Lambda_{n}$. Since $Q_{n \mid D, \varepsilon, \text { per }}$ is invariant under translations modulo $A_{n}, P^{(n)}(f)-Q_{n \mid D, c, \text { per }}(f) \rightarrow 0$ as $n \rightarrow \infty$, for all $f \in \mathcal{L}$; cf. Lemma 4.6 of [8]. Just as in Step 3) of the proof of Lemma 5.1 we obtain

$$
I\left(P^{(n)}\right) \leqq v_{n}^{-1} I\left(Q_{n \mid D, \varepsilon, \text { per }} ; Q_{n}\right)=-v_{n}^{-1} \log Q_{n}\left(\rho\left(R_{n}\right) \in D, \Phi\left(R_{n}\right) \leqq \varepsilon\right) .
$$

Lemma 7.2 thus implies that

$$
\lim _{n \rightarrow \infty} \sup I\left(P^{(n)}\right) \leqq-s(D, \varepsilon)<\infty .
$$

Since $I$ has compact (and sequentially compact) level sets, it follows that the sequence $\left(P^{(n)}\right)_{n \geqq 0}$, and thus also the asymptotically equivalent sequence $\left(Q_{n \mid D, e, p e r}\right)_{n \geqq 0}$, are relatively compact. Moreover, each accumulation point of any of these sequences belongs to the set $\{I \leqq-s(D, \varepsilon)\}$. On the other hand, Lemma 5.7 of [10] asserts that $P^{(n)}$ is asymptotically equivalent to

$$
Q_{n \mid D, \varepsilon, \mathrm{per}} R_{n} \equiv \int Q_{n \mid D, \varepsilon, \mathrm{per}}(d \omega) R_{n, \omega}
$$

as $n \rightarrow \infty$, and the latter measures all belong to the closed convex set $\{\rho \in$ $\bar{D}, \Phi \leqq \varepsilon\}$, as is easily seen by approximating $Q_{n \mid D, \varepsilon, \text { per }}$ by suitable discrete measures. This proves the proposition.

To deduce the first part of (2.32) from Proposition 7.3 we choose a tangent plane $\left(v, \varepsilon^{\prime}\right) \rightarrow p+\beta \varepsilon^{\prime}-v \log z$ to the concave function $s(\cdot, \cdot)$ on the subset of $\left.\left.\bar{D} \times\right]-\infty, \varepsilon\right]$ on which $s(\cdot, \cdot)$ attains its maximum $s(D, \varepsilon)$. The monotonicity of $s(v, \cdot)$ implies that $\beta \geqq 0$. It then follows that, for all $P \in \mathcal{P}_{\Theta}$ with $\Phi(P)<\infty$,

$$
I(P) \geqq-s(\rho(P), \Phi(P)) \geqq-p-\beta \Phi(P)+\rho(P) \log z
$$

with equality when $P \in M_{D, \varepsilon}$. Since $M_{D_{, \varepsilon}} \neq \emptyset$, we may conclude that $p=p(z, \beta)$ and

$$
M_{D, \varepsilon} \subset\left\{I_{z, \beta}=0\right\} .
$$


Together with Proposition 7.3, this gives the first part of (2.32). The second part is the subject of the next proposition.

Proposition 7.4 For all $z, \beta>0$, the sequence $\left(P_{n, z, \beta, p e r}\right)_{n \geqq 0}$ is relatively compact, and all its accumulation points belong to $\left\{I_{z, \beta}=0\right\}$.

Proof. We proceed just as in the proof of the last proposition. For each $n \geqq 0$ we have

$$
\begin{aligned}
-v_{n}^{-1} \log Z_{n, z, \beta, \mathrm{per}}= & v_{n}^{-1} I\left(P_{n, z, \beta, \mathrm{per}} ; Q_{n}\right) \\
& +\beta P_{n, z, \beta, \mathrm{per}}\left(\Phi\left(R_{n}\right)\right)-P_{n, z, \beta, \mathrm{per}}\left(v_{n}^{-1} N_{n}\right) \log z .
\end{aligned}
$$

The first term on the right-hand side is not less than $I\left(P^{(n)}\right)$, where $P^{(n)}$ is the invariant $\Lambda_{n}$-average of the $\Lambda_{n}$-periodic measure $\hat{P}^{(n)}$ making the configurations in the blocks $A_{n}+(2 n+1) i, i \in L$, independent with identical distribution $P_{n, z, \beta, \text { per }}$. Using Lemma 3.3 and the observation that $P_{n, z, \beta \text {,per }}\left(\rho^{(2)}\left(R_{n}\right)\right)=\rho^{(2)}\left(P_{n, z, \beta, \text { per }}\right)<\infty$ we see further that the second term on the right-hand side is not less than $\beta \Phi\left(P_{n, z, \beta \text {, per }} R_{n}\right)$. Combining this with (2.22) we obtain

$$
\limsup _{n \rightarrow \infty}\left[I\left(P^{(n)}\right)+\beta \Phi\left(P_{n, z, \beta, p e r} R_{n}\right)-\rho\left(P^{(n)}\right) \log z\right] \leqq-p(z, \beta)<\infty .
$$

The compactness of the level sets of $I$, the lower semicontinuity of $\Phi$, and the asymptotic equivalence of $P^{(n)}, P_{n, z, \beta, p e r} R_{n}$, and $P_{n, z, \beta, \text { per }}$ thus give the result.

To conclude, we mention without proof that the entropy function $s(\cdot, \cdot)$ is related to the pressure by the classical Legendre transformation as follows. For all $z, \beta>0$,

$$
p(z, \beta)=\max _{\nu \geq 0, \varepsilon \in \mathbf{R}}[s(v, \varepsilon)-\beta \varepsilon+\nu \log z] .
$$

Conversely,

$$
s(v, \varepsilon)=\min _{\beta \geq 0, z>0}[p(z, \beta)+\beta \varepsilon-v \log z]
$$

whenever $v>0$ and $s(v, \varepsilon)>-\infty$.

Acknowledgement. I would like to thank Y. Suhov for bringing reference [6] to my attention, and an anonymous referee for reminding me of reference [3].

\section{References}

[1] Dembo, A., Zeitouni, O.: Large Deviations Techniques and Applications. Boston: Jones and Bartlett 1993

[2] Deuschel, J.-D., Stroock, D.W.: Large Deviations. Boston: Academic Press 1989

[3] Dobrushin, R.L.: Gibbsian random fields for particles without hard core. Theor. Math. Phys. 4, 705-719 (1970)

[4] Dobrushin, R.L., Minlos, R.A.: Existence and continuity of the pressure in classical statistical physics. Theory Probab. Appl. 12, 535-559 (1967)

[5] Fritz, J.: Generalization of McMillan's theorem to random set functions. Stud. Sci. Math. Hung. 5, 369-394 (1970)

[6] Gallavotti, G., Miracle-Sole, S.: A variational principle for the equilibrium of hard sphere systems. Ann. Inst. Henri Poincaré A 8, 287-299 (1968)

[7] Georgii, H.O.: Gibbs Measures and Phase Transitions. Berlin: de Gruyter 1988 
[8] Georgii, H.O.: Large deviations and maximum entropy principle for interacting random fields on $Z^{d}$. Ann. Probab. 21, 1845-1875 (1993)

[9] Georgii, H.O.: Large deviations for hard core particle systems. In: Kotecky, R. (ed.) Proceedings of the 1992 Prague Workshop on Phase Transitions. Singapore: World Scientific 1993

[10] Georgii, H.O., Zessin, H.: Large deviations and the maximum entropy principle for marked point random fields. Probab. Theory Relat. Fields 96, 177-204 (1993)

[11] Lanford, O.E.: Entropy and equilibrium states in classical statistical mechanics. In: Lenard, A. (ed.) Statistical Mechanics and Mathematical Problems. (Lect. Notes Phys. 20) Berlin Heidelberg New York: Springer 1973

[12] Martin-Löf, A.: Statistical Mechanics and the Foundations of Thermodynamics. (Lect. Notes Phys. 101) Berlin Heidelberg New York: Springer 1979

[13] Matthes, K., Kerstan, J., Mecke, J.: Infinitely Divisible Point Processes. Chichester: Wiley 1978

[14] Nguyen X.X., Zessin, H.: Ergodic theorems for spatial processes. Z. Wahrscheinlichkeitstheor. Verw. Geb. 48, 133-158 (1979)

[15] Olla, S., Varadhan, S.R.S., Yau, H.T.: Hydrodynamic limit for a Hamiltonian system with a weak noise. Commun. Math. Phys. 155, 523-560 (1993)

[16] Ruelle, D.: Classical statistical mechanics of a system of particles. Helv. Phys. Acta 36, 183-197 (1963)

[17] Ruelle, D.: Statistical Mechanics. Rigorous results. New York: Benjamin 1969

[18] Ruelle, D.: Superstable interactions in classical statistical mechanics. Commun. Math. Phys. $18,127-159(1970)$ 